\title{
A high efficiency superconducting nanowire single electron detector
}

Cite as: Appl. Phys. Lett. 97, 183106 (2010); https://doi.org/10.1063/1.3506692

Submitted: 11 July 2010 . Accepted: 05 October 2010 . Published Online: 03 November 2010

M. Rosticher, F. R. Ladan, J. P. Maneval, S. N. Dorenbos, T. Zijlstra, T. M. Klapwijk, V. Zwiller, A. Lupaşcu, and G. Nogues

\section{ARTICLES YOU MAY BE INTERESTED IN}

Picosecond superconducting single-photon optical detector

Applied Physics Letters 79, 705 (2001); https://doi.org/10.1063/1.1388868

Efficient single particle detection with a superconducting nanowire

AIP Advances 2, 032124 (2012); https://doi.org/10.1063/1.4740074

Single-photon detectors combining high efficiency, high detection rates, and ultra-high timing resolution

APL Photonics 2, 111301 (2017); https://doi.org/10.1063/1.5000001

\section{Challenge us.}

What are your needs for periodic signal detection?

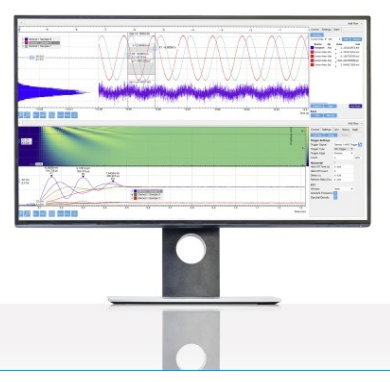




\title{
A high efficiency superconducting nanowire single electron detector
}

\author{
M. Rosticher, ${ }^{1}$ F. R. Ladan, ${ }^{1}$ J. P. Maneval, ${ }^{1}$ S. N. Dorenbos, ${ }^{2, a)}$ T. Zijlstra, ${ }^{2}$ T. M. Klapwijk, ${ }^{2}$ \\ V. Zwiller, ${ }^{2}$ A. Lupaşcu, ${ }^{3,4}$ and G. Nogues ${ }^{3,5}$ \\ ${ }_{1}^{1}$ Laboratoire Pierre Aigrain, CNRS, ENS, UPMC, 24 rue Lhomond, 75231 Paris Cedex 05, France \\ ${ }^{2}$ Kavli Institute of Nanoscience, Delft University of Technology, 2628CJ Delft, The Netherlands \\ ${ }^{3}$ Laboratoire Kastler Brossel, CNRS, ENS, UPMC, 24 rue Lhomond, 75231 Paris Cedex 05, France \\ ${ }^{4}$ Institute for Quantum Computing, University of Waterloo, 200 University Av. W, Waterloo, \\ Ontario N2L 3G1, Canada \\ ${ }^{5}$ Institut Néel/CNRS, Université Joseph Fourier, CEA-BP 166, 38042 Grenoble, France
}

(Received 11 July 2010; accepted 5 October 2010; published online 3 November 2010)

\begin{abstract}
We report the detection of single electrons using a $\mathrm{Nb}_{0.7} \mathrm{Ti}_{0.3} \mathrm{~N}$ superconducting wire deposited on an oxidized silicon substrate. While it is known that this device is sensitive to single photons, we show that it also detects single electrons with kilo-electron-volt energy emitted from the cathode of a scanning electron microscope with an efficiency approaching unity. The electron and photon detection efficiency map of the same device are in good agreement. We also observe detection events outside the active area of the device, which we attribute to sensitivity to backscattered electrons. (C) 2010 American Institute of Physics. [doi:10.1063/1.3506692]
\end{abstract}

The versatility of superconducting nanowires as single particle detectors relies on their sensitivity to the minute amount of energy required to locally induce a resistive transition. From this point of view, the latest achievements involving the detection of organic molecules ${ }^{1}$ and photons in the infrared range ${ }^{2}$ all derive from early experiments with $\alpha$-particles in the million electron volt range. ${ }^{3}$ In order to go beyond the optical resolution limit, the scanning electron microscope (SEM) working at low temperature, ${ }^{4}$ proved useful. This technique enabled the visualization of the real size of the hot spot caused by a detection process. ${ }^{5}$ However, the best achieved spatial resolution is limited by thermal diffusion to about $1 \mu \mathrm{m}$ and single electron detection has not been demonstrated. In this paper, we show single electron detection using a superconducting nanowire. Our superconducting single electron detector (SSED) offers a high spatial and timing resolution and we compare the electron detection efficiency map with a photon detection efficiency map, measured on the same device.

The fabrication process of our superconducting nanowire has been described before. ${ }^{6}$ It consists of a $100 \mathrm{~nm}$ wide, $500 \mu \mathrm{m}$ long, and $6 \mathrm{~nm}$ thick wire of $\mathrm{Nb}_{0.7} \mathrm{Ti}_{0.3} \mathrm{~N}$. The wire is folded into an $10 \times 10 \mu \mathrm{m}^{2}$ area, with a separation of $100 \mathrm{~nm}$ between adjacent detecting branches. One end of the wire is grounded whereas the other end is connected to a cryogenic coaxial cable used to inject a current through the structure. We measure a dc critical current $I_{c}=10 \mu \mathrm{A}$ at $4.2 \mathrm{~K}$ and $I_{c}=5.2 \mu \mathrm{A}$ at $8 \mathrm{~K}$. Our experimental setup consists of a cryogenic SEM. ${ }^{7}$ The detector is mounted on a cold translation stage at $\mathrm{T}=8 \mathrm{~K}$ under the electronic beam of an SEM. The SEM current $I_{b}$ is controlled and can be measured with a picoammeter (measurement uncertainty 10\%). The energy of the incident electrons $E_{e}$ can be varied between 5 and $30 \mathrm{keV}$.

To block low frequency $1 / f$ noise we use a dc-block, through which we can only bias the wire with pulses of current amplitude $I$ and duration $t_{d}=800$ ns (see inset of Fig. 1). Each pulse is reflected by the circuit. When the current is on,

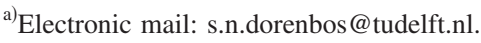

the detection of an electron triggers a short pulse at the output of the system (duration of a few nanoseconds, see inset of Fig. 1). The change in baseline is caused by the limited bandwidth $(0.1$ to $1000 \mathrm{MHz})$ of the amplification of the output pulses but does not affect the bias current of the SSED. We count the average number of detection pulses and compare it to the average incoming number of electrons $n_{i}$ $=I_{b} \times t_{d} / e$ in order to infer the absolute detection efficiency $\eta$. We can adjust the current $I_{b}$ down to a few picoamperes, and obtain single electrons separated by approximately 100 ns. We have measured the number of detection events $n_{d}$ versus incoming electrons $n_{i}$ (Fig. 1). The linearity of this plot shows that we detect single electrons. A complete map of $\eta$ is obtained by scanning the electron beam over the sample with a full control of the step size and dwell time.

To compare the relative performances of our superconducting nanowire detector for single electrons and single photons, we present in Fig. 2(a) a two-dimensional (2D) plot of the photon detection efficiency for our sample, obtained by scanning a laser spot $(1 \mu \mathrm{m}$ diameter $)$ over the detector with an intensity, low enough to be in the single photon

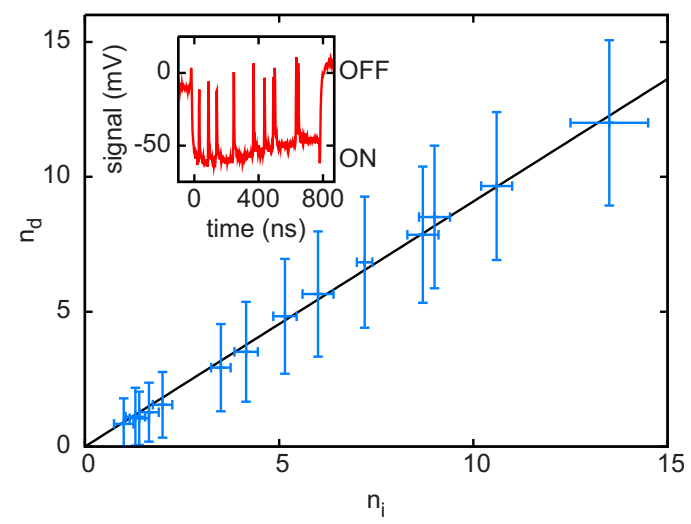

FIG. 1. (Color online) Average number of counted peaks per bias current pulse $n_{d}$ as a function of the number of incoming electrons $n_{i}=I_{b} \times t_{d} / e$ $\left(E_{e}=10 \mathrm{keV}, I / I_{c}=0.85\right)$. A linear fit (solid line) gives a detection efficiency $\eta=0.91(0.01)$. (Inset) A typical experimental signal at the output of the detection chain. One pulse corresponds to the detection of one electron. 
(a)

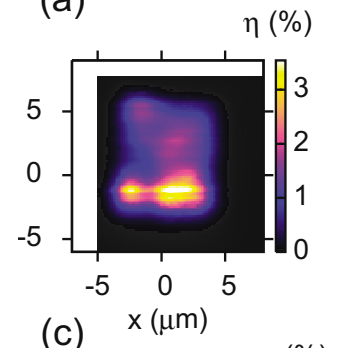

(c) $x(\mu \mathrm{m}) \quad \eta(\%)$

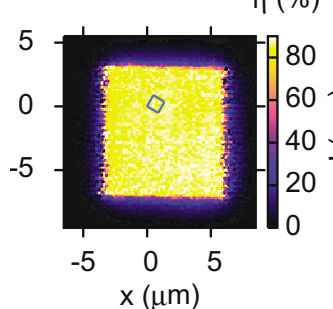

(b)

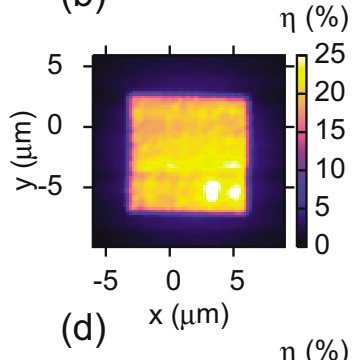

(d)

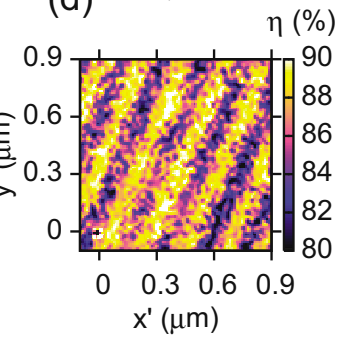

FIG. 2. (Color online) 2D plots of the detection efficiency. (a), for single photons $\left(\lambda=532 \mathrm{~nm}, I / I_{c}=0.8\right.$, step size $\left.\sim 100 \mathrm{~nm}\right)$. The white area corresponds to missing data (b), for single electrons (bias current $I / I_{c}=0.35$, electron energy $E_{e}=30 \mathrm{keV}$, SEM step size $200 \mathrm{~nm}$ ). (c), same map as (b) with $I / I_{c}=0.85, E_{e}=20 \mathrm{keV}$ and SEM step size $200 \mathrm{~nm}$. (d) Zoom of (c) for a $1 \times 1 \mu \mathrm{m}^{2}$ area, with SEM step size of $20 \mathrm{~nm}$. A square shown in (c) indicates the relative scale and orientation of $(\mathrm{d})$.

regime. A detailed description of the setup is given in Ref. 8. The map shows spatial variations over the active area that could originate from structural and/or chemical inhomogeneities of the layer. This reference image can be compared to Fig. 2(b) which shows the detection efficiency for electrons of energy $30 \mathrm{keV}$ and a detector bias current $I / I_{c}=0.35$. We observe that the electron detection efficiency is more homogeneous than in the case of photon detection. There exists however a clear correlation between the two measurements, with the maxima and minima located at the same positions for both images. It demonstrates that the detection process is affected in the same way for both particles. It shows also that SEM measurements could be used to characterize the performances of superconducting nanowires for photon detection. With increasing bias current, the electron detection efficiency saturates and becomes homogeneous over the whole detector active area as illustrated by Fig. 2(c) $\left(I / I_{c}\right.$ $\left.=0.85, E_{e}=20 \mathrm{keV}\right)$. We note that we have also been able to produce electron detection efficiency maps with $\mathrm{NbN}$ wires of 500 and $1000 \mathrm{~nm}$ widths. ${ }^{7}$ However, the present NbTiN detector covers a larger area and offers a better efficiency with fewer inhomogeneities.

In both Figs. 2(b) and 2(c), one observes sharper details than in Fig. 2(a). This is expected as electron microscopy has a much better spatial resolution than optical microscopy. In order to evaluate our spatial resolution, we present in Fig. 2(d) a $1 \times 1 \mu \mathrm{m}^{2}$ image of the detection efficiency taken in the conditions of Fig. 2(c). The plot directions make an angle of $60^{\circ}$ with respect to the wire direction. It reveals the inner structure of the detector via a small modulation of the detection efficiency ( 0.05 modulation, compared to an average efficiency of 0.85 ). The period of the modulation corresponds to the pitch of the meander structure and the visibility of this modulation indicates a spatial resolution of less than $100 \mathrm{~nm}$. We also observed that for electron energies $E_{e}$ $\leq 10 \mathrm{keV}$, the spatial resolution is degraded (to about 850 $\mathrm{nm}$ full width at half maximum) and one can no longer dis-

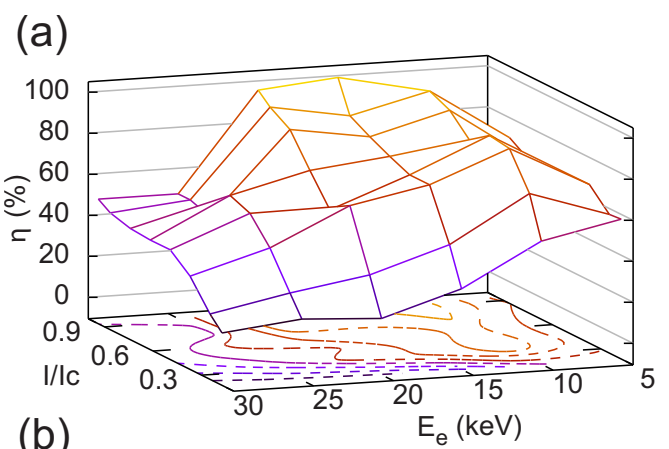

(b)

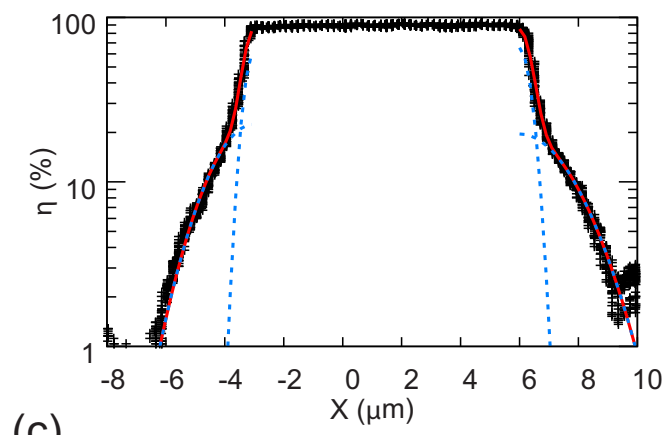

(c)

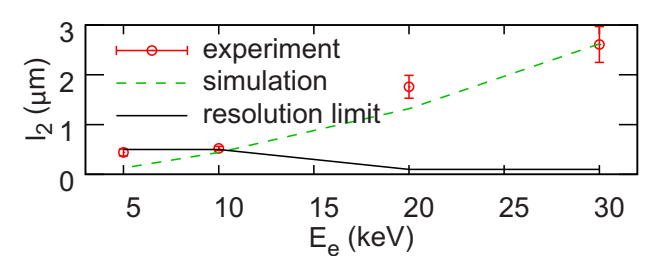

FIG. 3. (Color online) (a) Electron detection efficiency for different values of bias current and electron energy. The beam position is fixed and close to the center of the active area. (b) Cross-section of the detection efficiency map of Fig. 2(c) (log scale). The fall in detectivity at the edge of the active area is fitted by a double Gaussian (dotted lines: individual Gaussians, solid line: sum) with characteristic lengths $l_{1}=330 \mathrm{~nm}$ and $l_{2}=1760 \mathrm{~nm}$, respectively. (c) Plot of $l_{2}$ as a function of the electron energy $E_{e}$. At high energy $(\geq 10 \mathrm{kV})$, it is in good agreement with Monte Carlo simulations of the mean radius of exit for backscattered electrons, done in Ref. 12.

tinguish individual wires. We attribute this loss of performance to charging effects on the substrate which degrade the SEM spatial resolution.

Figure 3(a) summarizes our results for $\eta$ as a function of $I / I_{c}$ and $E_{e}$. It shows that $\eta$ increases with increasing $I / I_{c}$ and decreases with increasing electron energy. This fact is in good agreement with previous studies that showed that the mean electron energy loss $\Delta E_{e}$ is of the order or $100 \mathrm{eV}$ for the impinging electron energy range we have explored. ${ }^{9}$ Moreover, one has $\Delta E_{e}=S \times \lambda_{i n}$, where $\lambda_{i n}$ is the inelastic mean free path of the electron and $S$ is the stopping power of the material, which is well described by the Bethe formula ${ }^{10}$ or empirical variations of the latter. ${ }^{9,11}$ To a first approximation $S=f\left(E_{e}\right) / E_{e}$ and $\lambda_{i n}=g\left(E_{e}\right) \times E_{e}$ where $f$ and $g$ are slowly varying functions. As a consequence, the first inelastic collision in the detector happens deeper for high energy electrons and is less likely to trigger a transition of the nanowire, explaining the decrease in $\eta$ with increasing $E_{e}$. The low efficiency at $E_{e}=5 \mathrm{keV}(60 \%)$ is not consistent with this model. Our hypothesis is that it arises from trapped charges in the vicinity of the detector (i.e., in a dielectric or nonconductive material) close to the SSED that are also responsible for the degradation of the spatial resolution of the 2D-plots at this energy. 
Evidence of the detection of backscattered electrons is provided by the cross-section of the efficiency map of Fig. 2(c), presented in Fig. 3(b). We observe shoulders for the detection efficiency signal at the detector edges, proving that it is still possible to detect electrons impinging at distances larger than the SEM spatial resolution. A double Gaussian fit at the edge for different cross-sections, both horizontal and vertical, gives two average characteristic lengths $l_{1}$ $=330(40) \mathrm{nm}$ and $l_{2}=1760(230) \mathrm{nm}$, with a ratio in amplitude around 80:20. We attribute the first length to the finite step size of the SEM beam for this experiment. We attribute the second length scale to the detection of backscattered electrons. This distance is consistent with Monte Carlo simulations of the electron trajectories that predict a mean distance between the entry and exit points of $l_{\mathrm{MC}}=1320 \mathrm{~nm}$. This distance depends strongly on the energy of the incident electron. We present in Fig. 3(c) a comparison of $l_{2}$ and $l_{\mathrm{MC}}$ for different values of $E_{e}$. At low energy, the distance $l_{\mathrm{MC}}$ is of the order of or lower than the apparatus spatial resolution degraded to $500 \mathrm{~nm}$. We only observe a single Gaussian. At larger energies, above $10 \mathrm{keV}$, the agreement between experiment and simulation is very good. ${ }^{12}$

In conclusion, we have operated a fast and efficient single electron counter using a superconducting nanowire. We show an efficiency close to unity. Its low noise, short dead time, and high timing accuracy make this device interesting in situations where a cryogenic environment is available. Optimizing further these devices for the specific purpose of electron detection can certainly improve future performances. For example, a thin conducting layer on top of the nanowire can reduce the charging effect and improve the efficiency for low energy electrons $(\leq 5 \mathrm{keV})$. For high energy electrons, increasing the superconducting layer thickness will improve the performances. Fabricating the devices over a thin membrane would avoid backscattering. Arrays of such devices, could provide an imaging system for electrons with a time resolution below $60 \mathrm{ps}^{13}$ and a pixel size below $10 \mu \mathrm{m}$. Those figures could significantly improve the tech- nologies presently used for example in ultrafast electron microscopy. ${ }^{14}$

M.R., F.R.L., J.P.M., A.L., and G.N. thank the Japan Science and Technology Corporation (ICORP: "Quantum Entanglement") and the Région Ile de France (Cnano IdF project). A.L. acknowledges support from the European Union through a Marie Curie fellowship. S.D. and V.Z. acknowledge NWO for funding. We thank Reinier Heeres for help with the optical measurement. T.M.K. acknowledges NWO for funding through Grant No. 614.061.010.

${ }^{1}$ N. Zen, A. Casaburi, S. Shiki, K. Suzuki, M. Ejrnaes, R. Cristiano, and M. Ohkubo, Appl. Phys. Lett. 95, 172508 (2009).

${ }^{2}$ G. Gol'tsman, O. Minaeva, A. Korneev, M. Tarkhov, I. Rubtsova, A. Divochiy, I. Milostnaya, G. Chulkova, N. Kaurova, B. Voronov, D. Pan, J. Kitaygorsky, A. Cross, A. Pearlman, I. Komissarov, W. Slysz, M. Wegrzecki, P. Grabiec, and R. Sobolewski, IEEE Trans. Appl. Supercond. 17, 246 (2007).

${ }^{3}$ D. E. Spiel, R. W. Boom, and J. E. C. Crittenden, Appl. Phys. Lett. 7, 292 (1965).

${ }^{4}$ J. R. Clem and R. P. Huebener, J. Appl. Phys. 51, 2764 (1980).

${ }^{5}$ R. Gross and D. Koelle, Rep. Prog. Phys. 57, 651 (1994).

${ }^{6}$ S. N. Dorenbos, E. M. Reiger, U. Perinetti, V. Zwiller, T. Zijlstra, and T. M. Klapwijk, Appl. Phys. Lett. 93, 131101 (2008).

${ }^{7}$ A. Lupascu, A. Emmert, M. Brune, G. Nogues, M. Rosticher, J. P. Maneval, F. R. Ladan, and J. C. Villegier, Proceedings of the 2009 IEEE Toronto International Conference on Science and Technology for Humanity (TIC-STH), 2009, p. 1011. doi:10.1109/TIC-STH.2009.5444534

${ }^{8}$ S. N. Dorenbos, E. M. Reiger, N. Akopian, U. Perinetti, V. Zwiller, T. Zijlstra, and T. M. Klapwijk, Appl. Phys. Lett. 93, 161102 (2008).

${ }^{9}$ A. Jablonski, S. Tanuma, and C. J. Powell, Surf. Interface Anal. 38, 76 (2006).

${ }^{10} \mathrm{H}$. Bethe and J. Ashkin, Experimental Nuclear Physics (Wiley, New-York, 1953).

${ }^{11}$ D. C. Joy and S. Luo, Scanning 11, 176 (1989).

${ }^{12}$ D. Drouin, A. R. Couture, D. Joly, X. Tastet, V. Aimez, and R. Gauvin, Scanning 29, 92 (2007).

${ }^{13}$ M. G. Tanner, C. M. Natarajan, V. K. Pottapenjara, J. A. O'Connor, R. J. Warburton, R. H. Hadfield, B. Baek, S. Nam, S. N. Dorenbos, E. Bermudez Urena, T. Zijlstra, T. M. Klapwijk, and V. Zwiller, Appl. Phys. Lett. 96, 221109 (2010).

${ }^{14}$ W. E. King, G. H. Campbell, A. Frank, B. Reed, J. F. Schmerge, B. J. Siwick, B. C. Stuart, and P. M. Weber, J. Appl. Phys. 97, 111101 (2005). 